\title{
Lepton Flavour Universality at LHCb
}

\author{
Francesca Dordei*广 \\ INFN Sezione di Cagliari, Monserrato, Italy and European Organization for Nuclear Research \\ (CERN), Geneva, Switzerland \\ E-mail: francesca.dordeiecern.ch
}

Lepton Flavour Universality implies the equality of the coupling between the gauge bosons and the three families of leptons. Semileptonic and rare decays of $b$ quarks provide an ideal laboratory to test this property and to search for physics beyond the Standard Model. In these proceedings a review of the recent Lepton Flavour Universality tests performed using data collected by the LHCb experiment in 2011 and 2012 are presented.

The 20th International Workshop on Neutrinos (NuFact2018)

12-18 August 2018

Blacksburg, Virginia

\footnotetext{
* Speaker.

${ }^{\dagger}$ On behalf of the LHCb collaboration
} 


\section{Introduction}

Although the Standard Model (SM) has been very successful in describing existing data [1], some fundamental questions are still unanswered. This leads physicists to believe that the SM is not the final theory of particle physics. At the time of writing however, no new particles beyond those already present in the SM have been observed. Decays of hadrons containing $b$-quarks that have been collected at the LHC so far allow to test the CKM picture of flavour and CP violation with unprecedented sensitivity. In particular, tests of the so-called lepton flavour universality (LFU) using suppressed processes in the SM, which can also be achieved using such decays, are among the most powerful probes of possible effects beyond the SM, hereafter referred to as New Physics (NP). Indeed, in the SM the electroweak couplings of leptons to gauge bosons are assumed to be independent of their flavour. This property is known as lepton flavour universality. As a consequence, differences in the branching fractions of semileptonic and rare leptonic decays with $e, \mu$ or $\tau$ in the final state are expected to be only caused by phase space and helicity-suppressed contributions. However, several NP models predict non-SM bosons that do not couple equally to the different lepton families. Thus, deviations from the SM prediction would be a strong signature of NP.

During the Run 1 of the LHC, the LHCb experiment [2] has collected a large sample of beauty hadrons that corresponds to an integrated luminosity of $3 \mathrm{fb}^{-1}$ at $p p$ centre-of-mass energies of 7 and $8 \mathrm{TeV}$, respectively. In the following, an overview of the measurements performed by the LHCb collaboration using this dataset is presented.

\section{Lepton flavour universality in rare leptonic decays}

Rare $b \rightarrow s l^{+} l^{-}$transitions, where $l$ stands for $e$ or $\mu$ are flavour changing neutral current that are highly suppressed in the SM. The ratios

$$
R_{X}=\mathscr{B}\left(B \rightarrow X \mu^{+} \mu^{-}\right) / \mathscr{B}\left(B \rightarrow X e^{+} e^{-}\right),
$$

between $B$ decays to final states with muons and electrons, where $X$ is a hadron containing an $s$ - or a $d$-quark, are predicted to be very close to unity in the SM. From an experimental point of view, electrons and muons behave very differently in the $\mathrm{LHCb}$ detector. In particular, while the latter are characterised by a high reconstruction efficiency and a very clean signature, the former emit large amounts of bremsstrahlung, which imply a significant degradation of the resolution on the invariant dilepton mass, partially recovered by dedicated algorithms in the reconstruction software. Moreover, different levels of background contamination are present in the two channels, which implies substantial differences in the analysis. To minimise the effect of systematic uncertainties, at $\mathrm{LHCb}$ the measurement has been performed as a double ratio of branching fractions with the resonant channel $\mathscr{B}\left(B \rightarrow X J / \psi\left(l^{+} l^{-}\right)\right)$. LHCb Run 1 data has been used to perform the most precise measurements of $R_{K}$ and $R_{K^{*}}$, with $K^{*} \rightarrow K^{+} \pi^{-}$. The former is performed in the range $1 \mathrm{GeV}^{2} / c^{4}<q^{2}<6 \mathrm{GeV}^{2} / c^{4}$, while the latter is performed in two regions, namely $0.045 \mathrm{GeV}^{2} / c^{4}<q^{2}<1.1 \mathrm{GeV}^{2} / c^{4}$ and $1.1 \mathrm{GeV}^{2} / c^{4}<q^{2}<6 \mathrm{GeV}^{2} / c^{4}$, where $q^{2}$ is the dilepton invariant mass. The measured value of $R_{K}$ is $0.745_{-0.074}^{+0.090}$ (stat.) \pm 0.036 (syst.) [3], which is in tension with the SM at the $2.6 \sigma$ level. The measured values of $R_{K^{*}}$ [4] in the aforementioned 
regions are $0.66_{-0.07}^{+0.11}$ (stat.) \pm 0.03 (syst.) and $0.69_{-0.07}^{+0.11}$ (stat.) \pm 0.05 (syst.), with a tension with the SM prediction of $2.1-2.3 \sigma$ and $2.4-2.5 \sigma$, respectively.

\section{Lepton flavour universality in semileptonic decays}

The branching fraction ratio $R\left(D^{(*)}\right)=\mathscr{B}\left(B^{0} \rightarrow D^{(*)-} \tau^{+} v_{\tau}\right) / \mathscr{B}\left(B^{0} \rightarrow D^{(*)-} \mu^{+} v_{\mu}\right)$ represents a sensitive probe for LFU. A precise measurement of a $B$ decay involving $\tau$ leptons is challenging at a hadron collider due to the large background from partially reconstructed $B$ hadron decays that can mimic the signal topology. Moreover, the signal decay kinematics can not be fully constrained because of the neutrinos in the final state. In the LHCb analysis, the $\tau^{+}$and $D^{*-}$ are reconstructed through the decays $\tau^{+} \rightarrow \mu^{+} v_{\mu} \bar{v}_{\tau}$ and $D^{*-} \rightarrow \bar{D}^{0}\left(\rightarrow K^{+} \pi^{-}\right) \pi^{-}$. The ratio $R\left(D^{*}\right)$ is measured to be $0.336 \pm 0.027$ (stat.) \pm 0.030 (syst.) [5]. LHCb also performed another analysis, where the $\tau$ lepton is reconstructed through the $\tau^{+} \rightarrow \pi^{+} \pi^{-} \pi^{+}\left(\pi^{0}\right) \bar{v}_{\tau}$ decay. The result of the measurement is $0.291 \pm 0.019$ (stat.) \pm 0.026 (syst.) \pm 0.013 (ext.) [6], where the third uncertainty originates from the limited knowledge of the branching fraction of the normalization decay mode. All LHCb measurements are in good agreement with previous measurements from BaBar and Belle and combined together are about $4 \sigma$ larger than the SM predictions [8].

\section{Conclusions}

Analyses of semileptonic and rare leptonic decays of $b$-hadrons are providing interesting and quite consistent hints of lepton flavour universality violation, which deserve further investigation. The upcoming results from the LHC Run 2 data, along with the inclusion of new decay modes, will certainly lead towards a clear picture. Excellent prospects are moreover foreseen for the Upgrade II phase of $\mathrm{LHCb}$ [7], where an integrated luminosity of $300 \mathrm{fb}^{-1}$ will be collected.

\section{References}

[1] M. Tanabashi et al. [Particle Data Group], "The Review of Particle Physics", Phys. Rev. D 98, 030001 (2018).

[2] A. Alves Jr. et al. [LHCb Collaboration], "The LHCb detector at the LHC", JINST 3, S08005 (2008).

[3] R. Aaij et al. [LHCb Collaboration], "Test of lepton universality using $B^{+} \rightarrow K^{+} \ell^{+} \ell^{-}$decays", Phys. Rev. Lett. 113, 151601 (2014).

[4] R. Aaij et al. [LHCb Collaboration], "Test of lepton universality with $B^{0} \rightarrow K^{* 0} \ell^{+} \ell^{-}$decays", JHEP 08, 055 (2017).

[5] R. Aaij et al. [LHCb Collaboration], "Measurement of the ratio of branching fractions $\mathscr{B}\left(\bar{B}^{0} \rightarrow D^{*+} \tau^{-} \bar{v}_{\tau}\right) / \mathscr{B}\left(\bar{B}^{0} \rightarrow D^{*+} \mu^{-} \bar{v}_{\mu}\right)$ ", Phys. Rev. Lett. 115, 111803 (2015).

[6] R. Aaij et al. [LHCb Collaboration], "Measurement of the ratio of the $B^{0} \rightarrow D^{*-} \tau^{+} v_{\tau}$ and $B^{0} \rightarrow D^{*-} \mu^{+} v_{\mu}$ branching fractions using three-prong $\tau$-lepton decays", Phys. Rev. Lett. 120, 171802 (2018).

[7] R. Aaij et al. [LHCb Collaboration], "Physics case for an LHCb Upgrade II - Opportunities in flavour physics, and beyond, in the HL-LHC era", arXiv:1808.08865 [hep-ex].

[8] Y. Amhis et al. [HFLAV], "Averages of $b$-hadron, $c$-hadron, and $\tau$-lepton properties as of summer 2016", Eur. Phys. J. C 77, 895 (2017). 